\title{
Relevancia de fuentes nitrogenadas nuevas y regeneradas en la columna de agua en Bahía Magdalena (SO Península de Baja California), México
}

Relevance of new and regenerated nitrogen sources in the water column of Magdalena Bay (SW Baja California Peninsula), Mexico

\section{Rafael Cervantes-Duarte ${ }^{1,2}$, Silverio López-López ${ }^{1}$, Fernando Aguirre-Bahena ${ }^{1}$, Eduardo González-Rodríguez ${ }^{3}$ y Sonia Futema-Jiménez ${ }^{1}$}

\begin{abstract}
${ }^{1}$ Centro Interdisciplinario de Ciencias Marinas, CICIMAR-IPN, Av. Instituto Politécnico Nacional, s/n Col. Playa Palo de Santa Rita, A.P. 592, La Paz, BCS, C.P. 23096, México. Becarios de la COFAA-IPN, EDI. rcervan@ipn.mx

${ }^{2}$ Instituto de Investigaciones Marinas (CSIC), Eduardo Cabello 6, 36208, Vigo, España

${ }^{3}$ Centro de Investigación Científica y de Educación Superior de Ensenada, CICESE, Unidad La Paz, Calle Miraflores 334, Fraccionamiento Bella Vista, A.P. 23050, La Paz, BCS, México

Abstract.- Six bimonthly surveys during 2007 were carried out in Magdalena Bay (Mexico) during spring tide conditions. Spatiotemporal variations of temperature, salinity, nitrite, nitrate, ammonium, phosphate and chlorophyll- $a$ were analyzed in the Bay. During spring-early summer (April-June) seawaters were colder and richer in new nutrients and chlorophyll- $a$ than autumn and winter (February and October-December). The coastal upwelling during spring-early summer contributes with new nutrients to the Bay, whereas bottom-recycled nutrients should be the main input during the autumn-winter period. Thus, nutrients availability, dissolved inorganic nitrogen and phosphate, promote a seasonal pattern of phytoplankton biomass in Magdalena Bay.
\end{abstract}

Key words: New nutrient, regenerated nutrient, chlorophyll-a, coastal upwelling

\section{INTRODUCCIÓN}

La zona costera de la península de Baja California se considera la región marina de mayor productividad biológica de México (Lluch et al. 2006). La elevada producción primaria, fundamental para el sostenimiento de sus recursos pesqueros (Álvarez et al. 1975, Bizarro 2008), es debida a una combinación favorable de procesos físicos y biológicos, como las surgencias costeras, que aportan nutrientes a la zona eufótica (Zaitsev et al. 2003). En esa costa suroccidental de la Baja California, el complejo lagunar Bahía Magdalena-Bahía Almejas, es considerado biológicamente un sistema altamente productivo y diverso, donde habitan especies tropicales, templadas y de transición (Lluch et al. 2006). Por ello es una importante zona pesquera en la Baja California tanto en términos de biomasa descargada (peces, crustáceos y moluscos) como en ingreso de divisas (Bizarro 2008). A pesar de su diversidad biológica y derrama económica, no se iniciaron las primeras investigaciones hidrográficas hasta las décadas de 1970-80 (Álvarez et al. 1975, Acosta \& Lara 1978, Guerrero et al. 1988), las cuales se ampliaron posteriormente (Cervantes et al. 2007, Robinson et al. 2007, Sánchez et al. 2007, Zaitsev et al. 2007, 2010, Cervantes et al. 2010).
Las condiciones oceanográficas en la bahía están influidas por los eventos de afloramiento en la zona costera contigua, la hidrodinámica mareal y los procesos biogeoquímicos en su interior (Cervantes et al. 2007, 2010, Robinson et al. 2007, Sánchez et al. 2007, Zaitsev et al. 2007, 2010). Sin embargo, no existen antecedentes acerca de la importancia de los nutrientes regenerados dentro de la bahía frente a los nutrientes 'nuevos' asociados al afloramiento (Dugdale \& Goering 1967). Estos procesos fertilizantes del agua litoral desempeñan un papel clave en la dinámica de la biomasa y producción primaria del fitoplancton. Por esa razón, los objetivos del presente estudio fueron: a) analizar la variación estacional de nutrientes (nitrito, nitrato, amonio y fosfato) en la columna de agua de la bahía y su relación con las condiciones hidrográficas costeras (temperatura y salinidad) y, b) interpretar las fluctuaciones en la biomasa de fitoplancton (estimada por la concentración de clorofila-a) en relación con la disponibilidad de nutrientes durante un ciclo anual (año 2007). 


\section{MATeRiales y MÉtodos}

\section{Área de estudio}

La Bahía Magdalena se encuentra ubicada en la costa occidental de la península de Baja California entre los $24^{\circ} 30^{\prime}-24^{\circ} 48^{\prime} \mathrm{N}$ y $111^{\circ} 51^{\prime}-112^{\circ} 09^{\prime} \mathrm{W}$ (Fig. 1), coordenadas que corresponden con el límite sur de la Corriente de California. La bahía tiene un área de $565 \mathrm{~km}^{2}$ y no recibe aportes fluviales; su profundidad máxima, $40 \mathrm{~m}$, se sitúa en la boca que la comunica con el océano. La zona litoral interna de la bahía que tiene menos de $10 \mathrm{~m}$ de profundidad y ocupa aproximadamente la mitad de su superficie, se caracteriza por la presencia de canales someros rodeados por extensas zonas de manglar. Al sur de la bahía existe un canal que comunica con la vecina Bahía Almejas. Las estaciones climáticas están determinadas por el ciclo de irradiación solar, los vientos dominantes y la época de tormentas tropicales. En invierno y principios de primavera se registran las menores temperaturas asociadas con vientos dominantes del Noroeste, mientras que en verano-otoño las temperaturas son las más elevadas como resultado de la influencia de vientos del Sur, propiciando la llegada de tormentas tropicales en esta zona. El régimen mareal es semidiurno, con una pleamar media superior de 0,8 $\mathrm{m}$ y una pleamar máxima de 1,5 m. La bahía está situada en una zona subtropical con un clima cálido-seco y precipitaciones entre 48 y $153 \mathrm{~mm}$ año $0^{-1}$, con variaciones estacionales de $71 \mathrm{~mm}$ entre otoño e invierno boreal (i.e., entre octubre y febrero) y $54 \mathrm{~mm}$ entre primavera y verano boreal (i.e., entre marzo y septiembre) (Sánchez et al. 2010).

\section{CAMPAÑas de MUESTREO}

Durante el 2007 se realizaron 6 campañas bimestrales desde febrero a diciembre, abarcando las estaciones climáticas de invierno-primavera (febrero, diciembre y abril) y verano-otoño (junio, agosto y octubre) a bordo de la embarcación CICIMAR-XXX en condiciones de mareas vivas; se muestrearon 14 estaciones localizadas en el interior de bahía Magdalena (Fig. 1). A fin de conocer la distribución termohalina en la columna de agua se consideró una sección dentro de la bahía paralela a la costa (Est. 5-11), además de estaciones someras (Est. 1-4 y 12) y estaciones junto a su boca (Est. 13 y 14). En cada estación de muestreo se obtuvieron perfiles verticales de temperatura y salinidad con un CTD Sea-Bird 19Plus y se recolectaron muestras de agua de superficie y fondo mediante botellas Niskin, General Oceanic de 5 L, para determinar las concentraciones de nutrientes (nitrito, nitrato, amonio y fosfato) y clorofila- $a$.

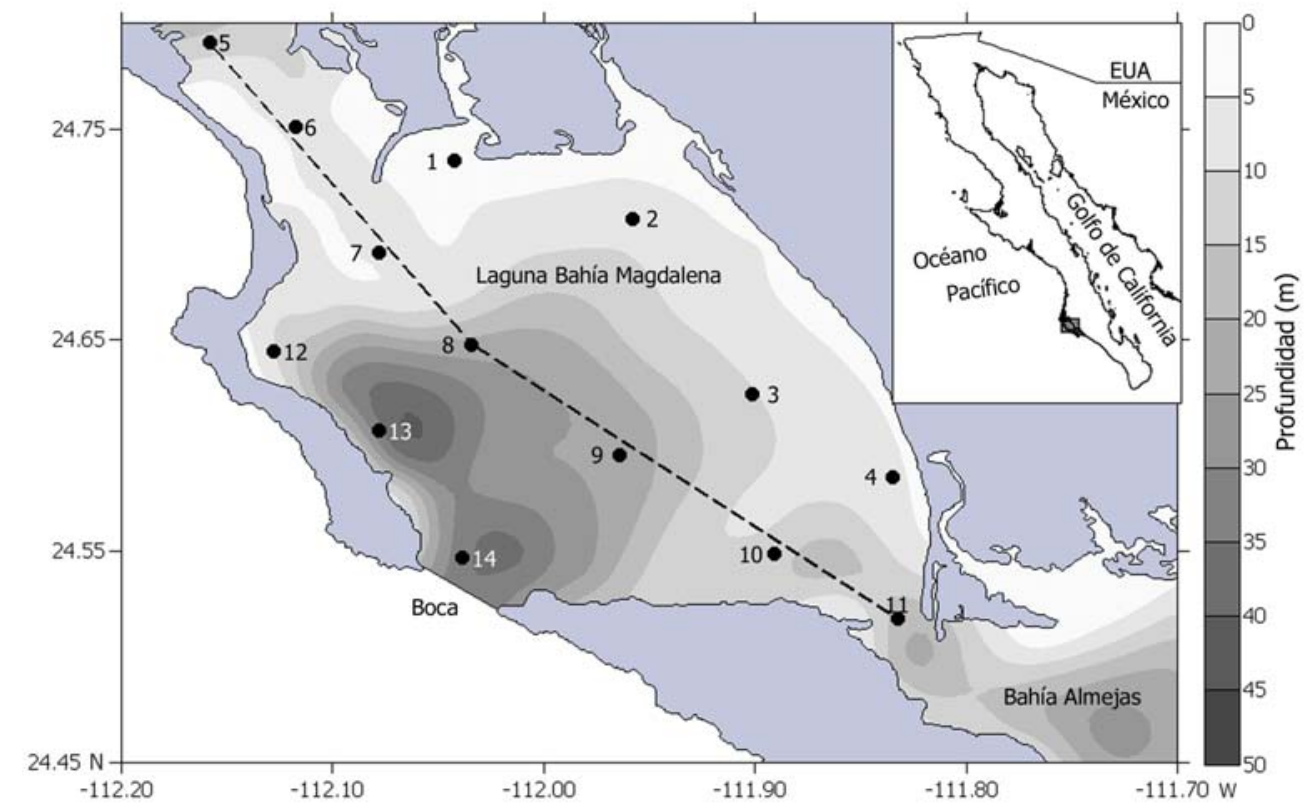

Figura 1. Localización geográfica del área de estudio y ubicación de las estaciones de muestreo en Bahía Magdalena / Geographic location of the study area and position of the sampling stations at Magdalena Bay 
Tratamiento de LAS MUESTRAS Y ANÁLISIS ESTAdÍstico

Para cada muestra se filtraron 2 L de agua en un sistema Millipore con filtros de fibra de vidrio Whatman GF/F de 25 mm de diámetro. La extracción de clorofila se realizó sin destruir el filtro de acuerdo con Venrick \& Hayward (1984) y la concentración de clorofila-a se estimó empleando las ecuaciones según el método descrito por Jeffrey \& Humphrey (1975), mediante un espectrofotómetro Spectronic ${ }^{\circledR}$ Genesys- ${ }^{\mathrm{TM}}$. Una parte del filtrado se utilizó para determinar la concentración de amonio, el cual se analizó dentro de las 6 h posteriores a la colecta. Otra parte se almacenó por un periodo de hasta $15 \mathrm{~d}$ en congelador a $-50^{\circ} \mathrm{C}$ para posteriormente analizar nitrito, nitrato y fosfato como ortofosfato. La determinación de las 4 sales nutrientes se realizó mediante técnicas espectrofotométricas estándares (Strickland \& Parsons 1972) usando un espectrómetro Spectronic $\AA^{\circ}$ Genesys-2 ${ }^{\mathrm{TM}}$.

A los datos de superficie y fondo de nitrógeno inorgánico disuelto (NID), fosfato y clorofila- $a$, se les aplicó la prueba de suma de rangos de Mann-Whitney, con el fin de averiguar si existen diferencias significativas entre estos niveles. Para identificar los periodos de relajación e intensificación del proceso de surgencia se consideraron los índices de surgencia costera diarios reportados por el Pacific Fisheries Environmental Laboratory ${ }^{1}$.

\section{RESUltados Y Discusión}

En aguas de Bahía Magdalena durante el periodo anual de 2007, la temperatura superficial varió entre16,4 y 26,4 $\mathrm{C}$ y la salinidad entre 33,5 y 37,6. La distribución de temperatura en superficie mostró un gradiente térmico desde la boca hacia el interior de la bahía, excepto a finales de invierno (febrero) que fue más homogénea (Fig. 2). Las menores temperaturas en la columna de agua se registraron en primavera y verano temprano $\left(14,2^{\circ} \mathrm{C}\right.$ en abril y $13,4^{\circ} \mathrm{C}$ en junio) junto a la boca. Zaitsev et al. (2010) señalaron para el periodo de 1996 a 2001 que las aguas superficiales dentro de la bahía fueron más cálidas, de abril a septiembre, que las aguas costeras adyacentes, debido a la surgencia marina. En 2007, se mantuvo este patrón en octubre y diciembre debido a las condiciones de flujo de la marea, circulación invernal dentro del sistema lagunar de Magdalena-Almejas y efecto de la Corriente de California (Morales et al. 2006). En invierno, específicamente en febrero, ocurrió lo contrario, es decir, la temperatura del agua al interior de la bahía fue inferior a la de la boca debido al enfriamiento invernal de la superficie marina.

${ }^{1}<$ http://www.pfeg.noaa.gov/pfel/about_pfel.html>

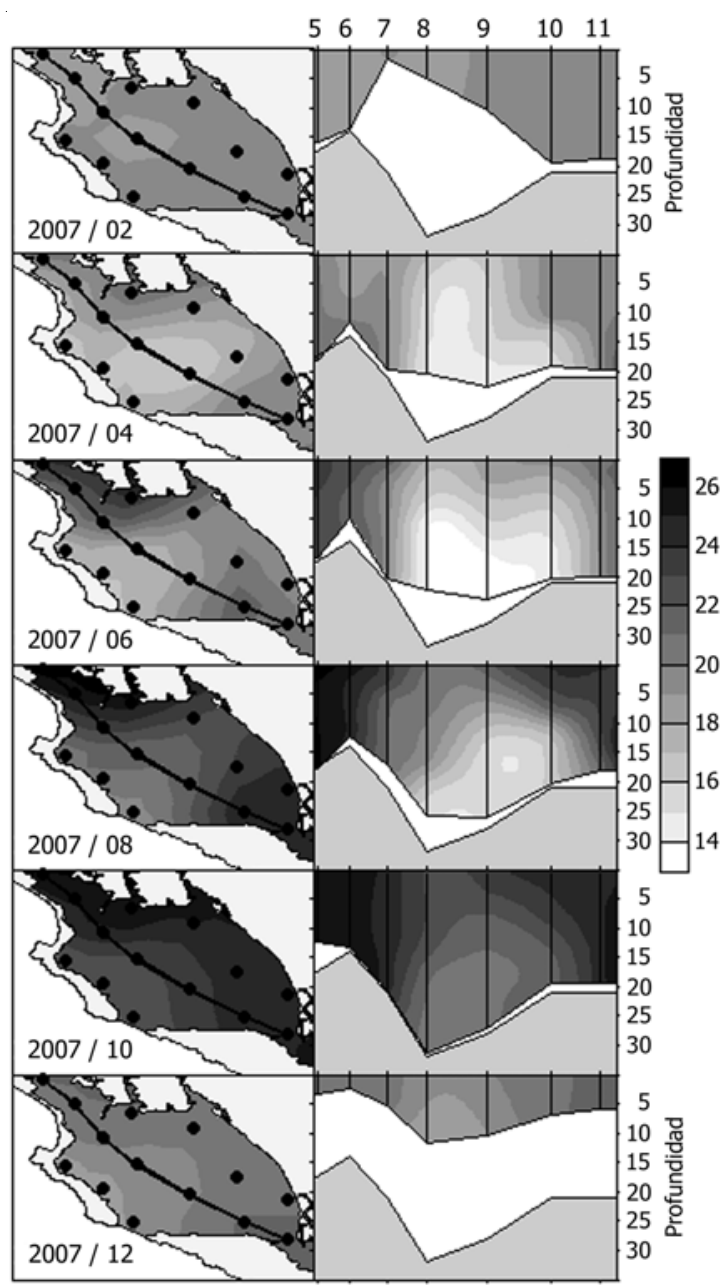

Figura 2. Distribución de temperatura superficial del mar (izquierda) y la columna de agua en la sección media (derecha) de bahía Magdalena durante el periodo de febrero a diciembre de 2007 / Sea surface temperature maps (left) and section 5-11 (right) of Magdalena Bay from February to December 2007

El nitrito presentó un rango de 0,01 a 0,81 $\mu \mathrm{M}$ (Fig. 3), siendo el nutriente con menor aporte al sistema, tal como había sido reportado anteriormente (Cervantes et al. 2007, 2010). Las muestras de fondo presentaron las mayores concentraciones, excepto en invierno (febrero y diciembre). El nitrato presentó una distribución similar al nitrito, aunque con un rango mayor de 0,1 a 20,4 $\mu \mathrm{M}$ (Fig. $3)$. Las mayores concentraciones se registraron en las muestras de fondo durante primavera y verano (abril, junio y agosto, respectivamente): Est. 14 (17,3; 15,4 y 15,8 $\mu \mathrm{M})$, Est. 13 (11,3; 9,9 y 50,0 $\mu \mathrm{M})$, Est. 9 (15,8; 17,5 y 17,3 $\mu \mathrm{M})$ y Est. $8(10,3 ; 15,9$ y 13,1 $\mu \mathrm{M})$. Estos máximos tuvieron lugar en la zona más profunda y cercana a la boca (Fig. 1). 

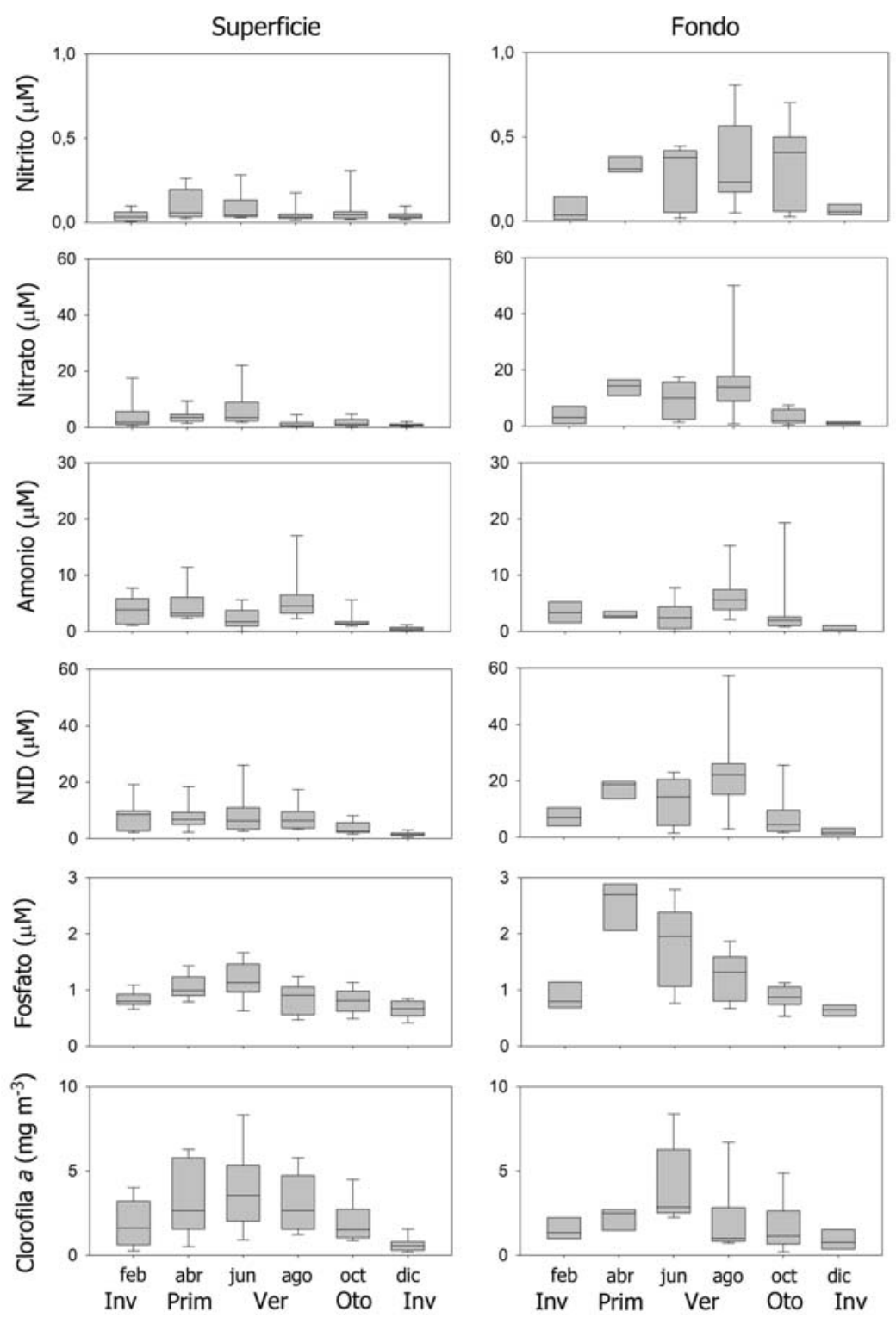

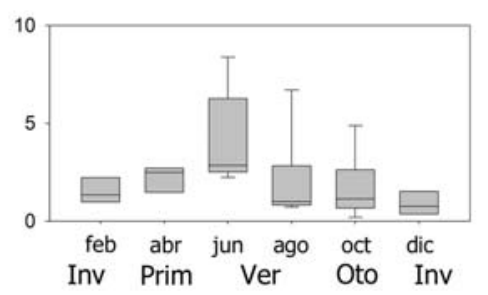

Figura 3. Concentración bimestral de nitrito, nitrato, amonio, nitrógeno inorgánico disuelto (NID), fosfato y clorofila- $a$ en superficie y fondo de bahía Magdalena. Se muestra la mediana y los percentiles del 5 y 95\%. Inv = invierno, Prim $=$ primavera, Ver $=$ verano y Oto $=$ otoño $/$ Bimonthly concentration of nitrite, nitrate, ammonium, dissolved inorganic nitrogen (DIN), phosphate, and chlorophyll- $a$ in surface and bottom waters of Magdalena Bay. The median and percentiles of 5th and 95th are shown. Inv = winter, Prim = spring, Ver = summer and Oto = autumn
Durante el resto del año se observó una disminución de nitrato con valores promedio inferiores a 5,3 $\mu \mathrm{M}$ en coincidencia con el periodo de surgencias costeras menos intenso en 2007 y que es común en otros años, tal como ha sido descrito por Álvarez et al. (1975), esto es, con un menor aporte de nitrógeno inorgánico nuevo.

En cuanto al nutriente reciclado, las concentraciones de amonio presentaron un rango de 0,1 a 19,3 $\mu \mathrm{M}$ (Fig. 3). Su distribución temporal (superficie y fondo) no mostró un patrón estacional, sin embargo, las mayores concentraciones (mediana) se observaron en verano (agosto: 4,5 y 5,6 $\mu \mathrm{M}$, respectivamente) y las menores a principios de invierno (febrero: 0,4 y 0,2 $\mu \mathrm{M}$, respectivamente) (Fig. 3). Al igual que los otros nutrientes nitrogenados, el amonio presentó altos valores en superficie y en puntos localizados de la bahía: Est. 14 $(13,4 \mu \mathrm{M})$ en abril, Est. $5(15,2 \mu \mathrm{M})$ y Est. $9(18,8 \mu \mathrm{M})$ en agosto; mientras que en el fondo se localizaron hacia el norte de la bahía, Est. 5 (7,8 $\mu \mathrm{M})$ en junio, Est. $6(15,6 \mu \mathrm{M})$ en agosto y Est. $7(19,3 \mu \mathrm{M})$ en octubre. Esta zona es abundante en fanerógamas, como Zostera marina, que además es un hábitat de invertebrados que interviene en el reciclado de materia orgánica, lo que incrementa la concentración de amonio (Parsons et al. 1984). 
El fosfato ó ión ortofosfato es la fracción principal que resulta de la determinación de fósforo reactivo en agua de mar filtrada (Strickland \& Parsons 1972), cuya calibración demostró ser apropiada para este tipo de aguas naturales, en términos de posible interferencia con otros iones. Presentó un rango de 0,4 a 2,9 $\mu \mathrm{M}$, mostrando las mayores concentraciones promedio en el fondo, en la estación de primavera y verano temprano: abril $(2,5 \mu \mathrm{M})$ y junio $(1,8 \mu \mathrm{M})$ (Fig. 3). De acuerdo a la proporción en que son asimilados los nutrientes N:P, razón de Redfield, el nitrógeno es agotado antes que el fósforo, por lo que éste no debe considerarse un nutriente limitante para la producción primaria en la bahía (Cervantes et al. 2010). Las zonas someras fueron más ricas en este nutriente y sus concentraciones no sólo serían un resultado de los procesos de remineralización litorales, sino también consecuencia de la mineralogía costera: en el complejo lagunar Magdalena-Almejas hay una influencia natural de minerales fosfatados provenientes de fosforitas y de rocas metamórficas fosfatadas en la isla Margarita (Rodríguez et al. 2007).

La variación temporal de la suma de nutrientes nitrogenados (NID) y fosfato, en el agua superficial y de fondo en Bahía Magdalena fue similar, es decir hubo aumento de nutrientes en primavera y verano (abril, junio y agosto) (Fig. 3). No obstante, en la columna de agua las concentraciones de nutrientes cerca del fondo fueron significativamente mayores con respecto de las de superficie (NID, $P=0,014$; fosfato, $P=0,018$ ), especialmente en primavera y verano (Fig. 3). Este incremento de nutrientes cercano al sedimento puede asociarse con 2 procesos: uno oceanográfico, las surgencias costeras, y otro biogeoquímico, la remineralización de la materia orgánica. En ese sentido, los índices de surgencia aumentaron durante primavera y verano temprano: de abril a junio de $2007\left(1630-2230 \mathrm{~m}^{3} \mathrm{~s}^{-1} \mathrm{~km}^{-1}\right)$, mientras que la remineralización de materia orgánica, según las concentraciones de amonio indican que, excepto en invierno temprano (diciembre), este nutriente reciclado se encuentra disponible todo el año, principalmente hacia finales de verano, que es cuando ocurre un relajamiento de la surgencia entre julio y agosto de 2007 (500-820 m $\mathrm{s}^{-1} \mathrm{~km}^{-1}$ ).

La concentración de clorofila- $a$ superficial y de fondo durante el 2007, varió entre 0,1 y $10,7 \mathrm{mg} \mathrm{m}^{-3}$ (valor extremo) y 0,2 y $8,4 \mathrm{mg} \mathrm{m}^{-3}$, respectivamente; sin embargo, los valores medios (superficie y fondo) más altos se observaron durante primavera y verano temprano: abril (2,6 y 2,5 $\mathrm{mg} \mathrm{m}^{-3}$ ) y junio (3,5 y 2,8 $\mathrm{mg} \mathrm{m}^{-3}$ ) (Fig. 3). Este máximo estacional destaca frente a las bajas concentraciones medias obtenidas en invierno temprano: diciembre (0,6 y 0,8 $\left.\mathrm{mg} \mathrm{m}^{-3}\right)$ y el máximo coincide con los altos contenidos en clorofila- $a$ reportados en mayo de 2002 (Cervantes et al. 2007). Las concentraciones de clorofila no presentaron diferencias significativas entre superficie y fondo de la columna de agua en la bahía ( $\mathrm{N}$ = $84, \mathrm{U}=1693, P=0,572$ ) de manera similar a lo observado por Gárate et al. (2001) con la abundancia de fitoplancton. Este máximo de clorofila corresponde con una mayor disponibilidad de nutrientes en el agua (Fig. 3).

La Bahía Magdalena es una laguna costera sujeta a una alta explotación de recursos marinos, como moluscos, crustáceos y peces. La elevada biomasa extraída anualmente, solo puede ser compensada con una alta tasa de producción primaria, la cual debe ser soportada por una fuente externa de nutrientes. Dado que no se producen aportes fluviales, en esta región las surgencias costeras externas a la laguna cumplen un rol de gran importancia, generando un incremento en la concentración de nutrientes que luego son transportados al interior de la laguna por las corrientes de marea. Aunque los procesos de surgencia se presentan durante todo el año, el periodo de mayor ocurrencia e intensidad se presenta en primavera y verano temprano (abril y junio) (Zaitsev et al. 2003) coincidente con el incremento en nitrato (fuente nitrogenada alóctona). Durante el resto del año, y principalmente en agosto, se recicla parte de la materia orgánica fotosintetizada, lo cual se refleja en el incremento de la concentración de amonio (fuente nitrogenada regenerada).

La disponibilidad de nutrientes y las condiciones favorables para su utilización, promueven un patrón estacional en la biomasa de fitoplancton estimada por la concentración de clorofila- $a$, generando una variación estacional con un máximo en la estación de primavera y verano temprano.

\section{Agradecimientos}

Los autores expresamos nuestro agradecimiento a las constructivas sugerencias de tres evaluadores anónimos y del Dr. Ricardo Prego que nos ayudaron a mejorar nuestro manuscrito. El presente trabajo se realizó con el apoyo del Instituto Politécnico Nacional a través del proyecto SIP-20070265. Rafael Cervantes-Duarte recibió apoyo de CONACyT (169030) durante 2012 para Año Sabático y actualmente es becario de la COFAA-IPN y EDI. 


\section{LITERATURA CITADA}

Acosta J \& JR Lara. 1978. Resultados físicoquímicos en un estudio de variación diurna en el área central de Bahía Magdalena, BCS. Ciencias Marinas 5(1): 37-46.

Álvarez S, L Galindo \& A Chee. 1975. Características hidroquímicas de Bahía Magdalena, BCS. Ciencias Marinas 2(2): 94-110.

Bizarro J. 2008. A review of the physical and biological characteristics of the Bahia Magdalena lagoon complex (Baja California Sur, Mexico). Bulletin Southern California Academy of Sciences 107(1): 1-24.

Cervantes R, S López \& E González. 2007. Características hidrológicas de Bahía Magdalena, BCS, en el periodo 20012003. CICIMAR Oceánides, México 22(1-2): 1-11.

Cervantes R, S López, E González \& S Futema. 2010. Ciclo estacional de nutrientes, temperatura, salinidad y clorofila $a$ en Bahía Magdalena, BCS, México (2006-2007). CICIMAR Oceánides, México 25(2): 111-120.

Dugdale R \& J Goering. 1967. Uptake of new and regenerated forms of nitrogen in primary productivity. Limnology and Oceanography 12: 196-206.

Gárate I, G Verdugo \& D Siqueiros. 2001. Variations in phytoplankton assemblages during 1988-1989 in a subtropical lagoon system of the west coast of Mexico. Oceánides, México 16(1): 1-16.

Guerrero R, R Cervantes \& A Jiménez. 1988. Nutrient variation during a tidal cycle at the mouth of a coastal lagoon in the Northwest of Mexico. Indian Journal of Marine Science 17: 235-237.

Jeffrey S \& J Humphrey. 1975. New Spectrophotometric Equations for determining Chlorophylls a, b, c1 and c2 in algal phytoplankton and higher plants. Biochemie und Physiologie der Pflanzen 167:191-194.

Lluch D, S Hernández, E Balart, L Beltrán, P Del Monte, A González, S Lluch, A Navarrete, G Ponce, C Salinas, J López \& S Ortega. 2006. Desarrollo sustentable de la pesquería en México: Orientaciones Estratégicas, 436 pp. Centro de Investigaciones Biológicas del Noroeste/Senado de la República, México.

Morales M, A Aretxabaleta, F Werner \& S Lluch. 2006. Modelación de la circulación invernal y la retención de partículas en el sistema lagunar Bahía Magdalena-Almejas (Baja California Sur, México). Ciencias Marinas 32(4): 631647.

Parsons R, M Takahashi \& B Hargrave. 1984. Biological oceanographic processes, 330 pp. Pergamon Press, New Jersey.
Robinson J, J Gómez \& S Gómez. 2007. Efecto de la dinámica de las corrientes de marea en los organismos pelágicos en la boca de Bahía Magdalena. En: Funes R, J Gómez \& JR Palomares (eds). Estudios ecológicos en Bahía Magdalena, pp. 45-58. Centro Interdisciplinario de Ciencias Marinas, Instituto Politécnico Nacional, La Paz.

Rodríguez D, E Choumiline, L Méndez, B Acosta \& D Sapozhnikov. 2007. Composición química de los sedimentos y macroalgas del complejo lagunar MagdalenaAlmejas. En: Funes R, J Gómez \& JR Palomares (eds). Estudios ecológicos en Bahía Magdalena, pp. 63-84. Centro Interdisciplinario de Ciencias Marinas, Instituto Politécnico Nacional, La Paz.

Sánchez O, O Zaitsev \& M Saldivar. 2007. Condiciones hidrofísicas en el sistema lagunar Bahía Magdalena-Almejas. En: Funes R, J Gómez \& JR Palomares (eds). Estudios ecológicos en Bahía Magdalena, pp. 1-28. Centro Interdisciplinario de Ciencias Marinas, Instituto Politécnico Nacional, La Paz.

Sánchez A, E Choumiline, B López, S Aguiñiga, L Sánchez, A Romero \& D Rodríguez. 2010. Patrón de transporte de sedimento en Bahía Magdalena, Baja California Sur, México, inferido del análisis de tendencias granulométricas. Latin American Journal of Aquatic Research 38(2): 167-177.

Strickland D \& T Parsons. 1972. A practical handbook of seawater analysis. Bulletin of Fisheries Research Board of Canada 167: 1-310.

Venrick E \& T Hayward. 1984. Determining chlorophyll on the 1984 CALCOFI surveys. CalCOFI Reports 25: 74-79.

Zaitsev O, R Cervantes, O Sánchez \& A Gallegos. 2003. Coastal upwelling activity on the Pacific shelf of Baja California peninsula. Journal of Oceanography 59(4): 489502.

Zaitsev O, O Sánchez \& J Robinson. 2007. Características del ambiente hidrofísico de la plataforma continental y zona oceánica adyacente al sistema lagunar Bahía MagdalenaAlmejas. En: Funes R, J Gómez \& JR Palomares (eds). Estudios ecológicos en Bahía Magdalena, pp. 29-43. Centro Interdisciplinario de Ciencias Marinas, Instituto Politécnico Nacional, La Paz.

Zaitsev O, O Sánchez \& M Saldivar. 2010. Variaciones estacionales de la estructura termohalina en el sistema lagunar de Bahía Magdalena-Bahía Almejas y el mar adyacente. Ciencias Marinas 36(4): 413-432.

Recibido el 9 de marzo de 2012 y aceptado el 27 de agosto de 2012

Editor Asociado: Gabriela Muñoz C. 